\title{
Adrenocortical carcinoma markers
}

Important steps towards individualized medicine for adrenocortical carcinoma are revealed in two articles published by a German team. The researchers' identified GLUT1 as a powerful prognostic marker for adrenocortical carcinoma and ERCC1 as a predictive factor for response to platinum-based therapy in the disease.

Adrenocortical carcinoma is a rare disease with a poor prognosis. Unfortunately, this rarity has hampered the establishment of prognostic factors others than stage and resection status, and treatment is not standardized because randomized trial data are not available. Fassnacht and co-investigators recognized the need to identify molecular features of adrenocortical carcinomas that could provide useful information about prognosis and treatment response. Crucially, they had available more than 160 adrenocortical tumor samples from the German ACC Registry Group with which to provide the answers. "Prognostic markers are urgently needed to assist in the selection of patients for more aggressive therapy," explains researcher Martin Fassnacht of the University Hospital of Würzburg, Germany. "Furthermore, owing to the significant toxicity of aggressive treatment such a platinum-based chemotherapy, it would be a major step forward to identify in advance patients who are likely to respond to this therapy."

In a number of human carcinomas, key components of glucose metabolism such as GLUT1, GLUT3, TKTL1 and M2-PK are overexpressed and have been used as prognostic markers. To find out whether these four markers also have prognostic value in adrenocortical carcinoma, Fassnacht and co-researchers correlated clinical data with the results of immunohistochemical analysis of adrenocortical tumor tissue samples. Patients who had tumors with high GLUT1 expression had a considerably increased risk of death related to the disease than those with no GLUT1 expression. In addition, in multivariant analysis, the prognostic value of GLUT1 immunostaining was independent of tumor stage. By contrast, the expression of the three other markers of glucose metabolism did not correlate with clinical outcome. "Up to now, GLUT1 is (to our knowledge) the single factor with the best prognostic power in this rare disease," points out Fassnacht.

The second study, notes Fassnacht, was inspired by studies that have shown that expression of the DNA excision repair protein ERCC1 is a predictive factor for resistance to platinum-based chemotherapy in other tumor entities. The researchers used the same methodological approach as for the GLUT1 study to find out if the same is true in adrenocortical carcinoma. "The most exciting finding was that among 45 patients treated with platinum-based chemotherapy, those with low ERCC1 expression in their tumors had a statistically significant and clinically relevant prolonged survival time compared with those with high expression," comments Fassnacht "This is the first step towards an individualized medicine in this rare disease."

Fassnacht stresses that the findings of both studies need to be confirmed in independent cohorts before clinicians can start to use these markers. Fassnacht's team are, however, already using ERCC1 immunostaining to help predict treatment response in some patients.

\section{Carol Wilson}

Original articles Fenske, W. et al. Glucose transporter GLUT1 expression is an stage-independent predictor of clinical outcome in adrenocortical carcinoma. Endocr. Relat. Cancer 16, 919-928 (2009) | Ronchi, C. L. et al. Expression of excision repair cross complementing group 1 and prognosis in adrenocortical carcinoma patients treated with platinum-based chemotherapy. Endocr. Relat. Cancer 16, 907-918 (2009) 